\title{
Optimization of a $p$-persistent Network Diversity Multiple Access Protocol for a SC-FDE System
}

\author{
M. Pereira, Member, IEEE, L. Bernardo, Member, IEEE, R. Dinis, Member, IEEE, R. Oliveira, Member, IEEE, \\ P. Montezuma, Member, IEEE, and P. Pinto, Member, IEEE
}

\begin{abstract}
This paper presents a Medium Access Control (MAC) protocol solution designed to properly handle collisions when in the presence of a multi-packet detection receiver for Single-Carrier (SC) modulations with Frequency-Domain Equalization (FDE). It is considered an iterative frequencydomain receiver that jointly performs equalization, multi-packet separation and channel decoding operations, for up to $Q_{\max }$ mobile terminals transmitting in one slot. In this work, it is proposed and evaluated a $p$-persistent Network Diversity Multiple Access (NDMA) random MAC protocol designed to cope with a total number of mobile terminals $J$, for a maximum decoding capability of $Q_{\max }$ simultaneous packets. An accurate analytical model is presented to optimize two different scenarios: in the first one, a saturated network is considered and it is determined the packet transmission probability that maximizes the uplink throughput; the second represents a non-saturated network and the goal is to compute the optimal transmission probability associated to each mobile terminal that minimizes the packet transmission delay. In the end, analytical results obtained through physical and MAC layer simulations are discussed.
\end{abstract}

Index Terms-Multi-packet detection, k-limited network diversity multiple access (NDMA) protocol, $p$-persistent random access, cross-layer optimization.

\section{INTRODUCTION}

I $\mathrm{N}$ wireless systems that allow shared access multiple users might access a given channel, and the objective of the Medium Access Control (MAC) protocols is to allow this efficiently. A collision occurs when different users are simultaneously accessing the channel and the packets involved are too disrupted to allow their detection. Although this resembles the case where we have poor propagation conditions (e.g., due to shadowing effects), most of the appropriate countermeasures (taking advantage of the different degrees of freedom to improve the performance (modulation, transmit power, code rate, etc.) [1], [2], [3]) are not efficient to cope with collisions. The conventional approaches to cope with collisions are to discard all packets involved and to retransmit them, which corresponds to the conventional Automatic Repeat reQuest techniques (ARQ), or to avoid collisions using scheduled

Manuscript received June 3, 2011; revised January 11 and October 26, 2012; accepted September 25, 2013. The associate editor coordinating the review of this paper and approving it for publication was M. Torlak.

M. Pereira, R. Dinis, and R. Oliveira are with CTS, Uninova, Dep. ${ }^{o}$ de Eng. ${ }^{a}$ Electrotécnica, Faculdade de Ciências e Tecnologia, FCT, Universidade Nova de Lisboa, 2829-516 Caparica, Portugal and IT, Instituto de Telecomunicações, Portugal (e-mail: miguelpereira.pro@gmail.com, \{rdinis, rado\}@fct.unl.pt)

L. Bernardo, P. Montezuma, and P. Pinto are with CTS, Uninova, Dep. ${ }^{o}$ de Eng. ${ }^{a}$ Electrotécnica, Faculdade de Ciências e Tecnologia, FCT, Universidade Nova de Lisboa, 2829-516 Caparica, Portugal (e-mail: 1flb@fct.unl.pt, pmc@uninova.pt,pfp@fct.unl.pt).

Digital Object Identifier 10.1109/TWC.2013.110813.111056 transmissions. Collision free systems are commonly used with Hybrid ARQ/Forward Error Correction (FEC) strategies [4], which retain the signal associated to an erroneous packet and that may ask for additional redundancy, improving the systems' performance in the presence of fading and shadowing effects, eventually combining only the best received signals [5]. As an alternative, multi-packet detection mechanisms, relying on signals associated to multiple collisions (and the inherent diversity effects [6]), can be employed to separate the packets involved.

Separating multiple packets involved in a collision is essentially a multi-user detection problem [7]. To achieve the separation, we need multiple versions of each of the packets involved (at least $Q$ versions of each one if $Q$ users are involved in a collision). The traditional way of obtaining these multiple versions is by employing spread spectrum techniques. For instance, with DS-CDMA techniques (Direct Sequence Code Division Multiple Access) there is a multiplicity effect equal to the processing gain, which can be used in multiuser detection schemes [8]. However, this multiplicity effect is rigid and it needs to be set to the worst case scenario (above the highest number of users that we want to separate, in the case of CDMA systems), leading to waste of resources if the system is not fully loaded.

A multi-packet detection technique for flat fading channels was proposed in [9], where all users involved in a collision of $Q$ packets retransmit their packets $Q-1$ times. Therefore, the receiver has $Q$ versions of the signals associated to the $Q$ packets and jointly detects all packets involved ${ }^{1}$. To allow packet separation, different phase rotations are employed for different packet retransmissions. This technique was extended to multi-path time-dispersive channels in [10]. Since [10] considers a time-domain receiver implementation, its complexity can be very high for severely time-dispersive channels. Moreover, due to the linear nature of the receivers of [9] and [10], the residual interference levels can be high and/or we can have significant noise enhancement. Promising frequencydomain multi-packet detection schemes that allow efficient packet separation in the presence of successive collisions were proposed in [11][12]. These receivers are suitable to severely time dispersive channels and do not require uncorrelated channels for different retransmissions.

The MAC protocol employed on the systems described above is the Network Diversity Multiple Access (NDMA) and assumes that the receiver is always able to separate all

\footnotetext{
${ }^{1}$ This approach could be regarded as a "dynamic time-spreading" scheme, in opposition to conventional DS-CDMA techniques that could be denoted as "rigid spread spectrum" schemes.
} 
receiving packets regardless of the number of mobile terminals (MTs). If the number of MTs is large, the complexity for base stations (BS) may be too high. In a practical implementation the multi-packet receiver is able to handle a limited maximum number of packets $\left(Q_{\max }\right)$, which is much lower than the number of active MTs. Therefore, the MAC protocol must be designed to avoid having more than $Q_{\max }$ MTs transmitting packets simultaneously.

This paper considers a BS with a Multi-Packet Receiver (MPR) scheme based on [11][12] that is able to separate up to $Q_{\max }$ packets and it proposes a $p$-persistent Slotted ALOHA MAC protocol designed to cope with a $J$ number of MTs higher than $Q_{\max }$. This paper considers both exponential and constant backoff and it presents a suitable analytical model for the system's behavior for saturated load (when all nodes always have packets to transmit) and for homogeneous unsaturated load. The model takes the physical layer characteristics into account, namely the variation of the PER (Packet Error Rate) and packet transmission time with the number of packets involved in a collision, as well as packet retransmissions due to decoding errors. A half-duplex scenario is considered, where the throughput is influenced by the radio turnover and by the transmission and propagation times.

The major contribution of this paper is the design and evaluation of a $p$-persistent MAC protocol that extends NDMA to handle MPR with a number of MTs higher than $Q_{\max }$, with half-duplex radios and variable packet detection time. As far as we know, this paper is the first to propose a MAC protocol for these conditions. It proposes an analytical model to compute the network throughput and message delay for a variable detection time with different backoff algorithms. The access probability parameter, $p$ (equal for all MTs), that maximizes the saturation throughput, $p_{\text {sat }}{ }^{\star}$, is calculated and it is shown that for $p_{\text {sat }}{ }^{\star}$ the system allows high saturation throughput in noisy channels when a frequency-domain turbo multipacket reception is used. This paper also studies the average packet delay for finite exponential sources, and calculates the optimal access probability parameter $(p)$ that minimizes the packet delay, $p_{o p t}{ }^{\star}$.

The paper is organized as follows: an overview of related work is given in sec. II. The system's overview, including our multi-packet detection technique and the MAC protocol is presented in sec. III. The system's performance and the optimal configuration are analyzed in sec. IV and a set of performance results is presented in sec. V. Finally, sec. VI contains the conclusions.

\section{RELATED WORK}

A MAC layer properly tailored to the MPR characteristics of the physical layer has the potential of significantly improving the network performance [13]. A static channel slot assignment can be used to provide the optimal throughput for saturated load [14]. For variable load, undetectable collisions (involving more than $Q_{\max }$ MTs) can be avoided by applying a channel slot reservation mechanism which can be controlled either by the senders [15] or by the receivers [16]. Eisenberg et al. [15] assign a pair of RTS (Request To Send) / CTS (Clear To Send) bits to each MT, and the BS controls who transmits in the next data slot. This solution generates significant overhead when the number of MTs is large. In [16] the BS selects the set of transmitters for each slot, based on the information about their previous slot usage.

Another alternative is to let collisions happen, and then resolve them. The slotted ALOHA algorithm retransmits failed packets with a probability $p$. The stability condition for a finite-population ALOHA protocol in a CDMA network was analyzed in [17]. Naware et al. [18] studied its stability and delay over symmetric and asymmetric CDMA MPR channels. They proved the delay optimality and stability of 1-persistent ALOHA for MPR schemes able to separate all packets involved in a collision (like the ones proposed in [11][12] when the number of MTs is not above $Q_{\max }$ ). The ALOHA throughput, capacity and stability regions for spread spectrum techniques were studied in [19]. A finite-user Slotted ALOHA delay and throughput analysis was proposed in [20] for error free conditions. Samano-Robles et al. [21] studied the stability, throughput and delay properties under an imperfect detection assumption for NDMA, without considering a limiting $Q_{\max }$ value. Guo et al. [22] analyzed the capacity region of a CDMA MPR scheme with a finite $Q_{\max }$ value.

Several backoff algorithms were proposed to reduce the collision duration in MPR slotted MAC protocols [23][24]. The delay and jitter of IEEE 802.11 DCF (Distributed Coordinated Function), which uses exponential backoff, was evaluated in [23]. Celik et al. [24] proposed an alternative backoff mechanism that decreases the transmission probability after a success and increases it after a failure, improving the algorithm's fairness for heterogeneous distances to the receiver. Zhang [25] uses multiple 802.11 DCF RTS/CTS exchanges in multiple contention slots to optimize CDMA MPR, by maximizing the probability of having near $Q_{\max }$ users transmitting. Samano-Robles et al. [26] proposed a constant $p$-persistent access mode for NDMA considering a unlimited $Q_{\max }$ value.

Backoff algorithms previously proposed for the classical single-packet receivers (e.g. [27]: linear backoff, etc.) were also applied to the MPR scenario. The TA (Tree Algorithm) approach falls into the set of limited collision algorithms, which guarantees an upper-bounded transmission delay. In a TA, the set of contending MTs is reduced by half after a collision. Gau and Chen [28] studied the TA's throughput and delay for MPR and packet queues with capacity for one and two packets. Yu and Giannakis [29] proposed a SICTA approach, which combines a TA with a SIC scheme (Successive Interference Cancelation). In SICTA the collided packet signals are used to extract the individual packets and achieve a maximum stable throughput of 0.693. A simplified SIC approach was proposed by Yim et al. [30], denoted Dual Power Multiple Access (DPMA), where multipacket reception is achieved for a small number of users by carefully defining multiple power levels at the reception. Packets are decoded from the signal with the highest power to the lowest power, achieving a theoretical stable throughput higher than SICTA.

\section{SYSTEM OVERVIEW}

In this paper we consider the uplink transmission in structured wireless systems employing SC-FDE schemes where a 
set of $J$ MTs send data to a $\mathrm{BS}^{2}$. MTs are low resource battery operated devices whereas the BS is a resource rich device, which runs the multi-packet detection algorithm in real-time. MTs have a half-duplex radio and employ a $p$-persistent Slotted ALOHA algorithm to send data packets using the time slots defined by the BS (for the sake of simplicity, it is assumed that the packets associated to each user have the same length, $L_{\text {data }}$ ). The interaction between MTs and the BS has two phases: an association using a dedicated control channel, before the data transmission using the shared data channel. The BS uses the downlink channel to broadcast the MTs access probability ( $p_{c}$ for the $c$ th transmission attempt) and, possibly, to force packet retransmissions or block the transmission of new packets in the next slot. It is assumed that different data packets arrive simultaneously and perfect power control and time advance mechanisms exist, able to compensate a different attenuation and propagation times. We assume perfect channel estimation, user detection, and synchronization at the receiver side. Data packets are composed of $N_{F F T}$ FFT (Fast Fourier Transform) blocks and have a physical preamble overhead of $N_{\text {PhyPreamble }}$ symbols. Each FFT block carries $N_{\text {Block }}$ symbols. The physical preamble is used to estimate the channel, synchronize the reception and detect the users involved in a given collision.

\section{A. MAC protocol}

The uplink slots are organized as a sequence of epochs, where one or more groups of detection slots form an epoch. An illustration of the algorithm employed in the BS is depicted in Algorithm 1. The BS broadcasts a SYNC (Synchronization) control packet through the downlink channel marking the beginning of each epoch, allowing any MT with data packets to contend in the next slot (the first slot of the first group of detection slots) with equal access probability $p_{1}$ (i.e. assuring fairness). Other MTs that do not transmit, have to wait until the beginning of the next epoch.

If at least one MT transmits in the group of detection slots $c$, a signal is received by the $\mathrm{BS}$ and the $\mathrm{BS}$ detects the number of MTs' messages involved in the signal received. While this number is above the maximum bound allowed $\left(Q_{\max }\right)$, the BS detects that it is a collision but it cannot decode the signal. This is designated as an unintelligible collision, where the group of detection slots has a single slot. After an unintelligible collision, the BS broadcasts an ACK (Acknowledgment) packet defining a new value for the access probability $p_{c+1}$ for the next group of detection slots, which will exclude some MTs from transmitting during this epoch. Two backoff algorithms are considered:

- Constant backoff (CB): $p_{c}=p$;

- Exponential backoff (EB): $p_{c}=p^{c}$.

An epoch ends when the number of transmitting MTs in the group of detection slots $c, Q$, is equal to or below $Q_{\max }$. The BS broadcasts an ACK packet at the end of the slot with the value of $Q-1$ informing the MTs to retransmit the data packets, making possible the detection of the data packets. The data packets are retransmitted continuously (reducing the

\footnotetext{
${ }^{2}$ Our system design also allows the definition of an ad hoc operation mode, with MT to MT communication.
}

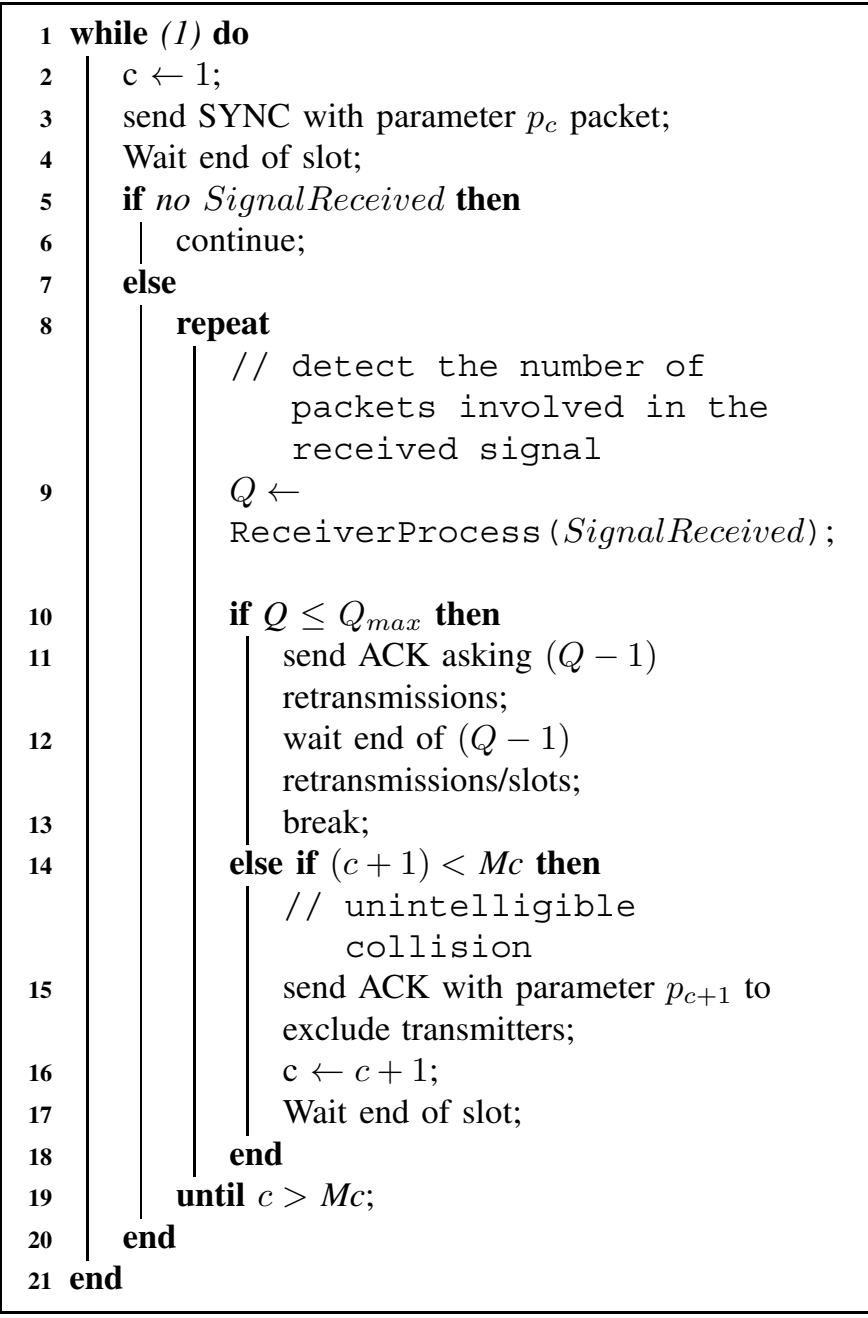

Algorithm 1: Algorithm at the Base Station

control overhead) and the last control packet sent by the BS is the SYNC one marking the end of this epoch and announcing the beginning of a new one. This SYNC packet carries a list of detected senders and a bit mask informing whether each of the packets was well received or not in the epoch ending.

An epoch also ends if the BS does not receive any signal during the first slot of the group of detection slots $c$ (i.e. no MT is transmitting), or when the maximum number of groups of detection slots, denoted as $M_{C}$, is reached. In these cases, no data packet is received at the BS.

Failed and excluded data packets are retransmitted in the next epochs up to a maximum number of times, denoted by $M_{R}$, being discarded after that.

Fig. 1 illustrates four possible situations that may occur during the MAC operation, with 3 MTs and $Q_{\max }=2$. It also contains the instants when new packets are ready to be sent. The first epoch was successful with 2 active senders. The second epoch was empty as no-one transmitted. The third epoch starts with a unintelligible collision and in its second slot one MT gave up and two proceeded. Both succeeded to transmit. The last epoch of the figure shows a successful transmission of a single MT.

Data, SYNC and ACK packets from different senders are spaced by a SIFS (Short Inter-Frame Space) time, to support 


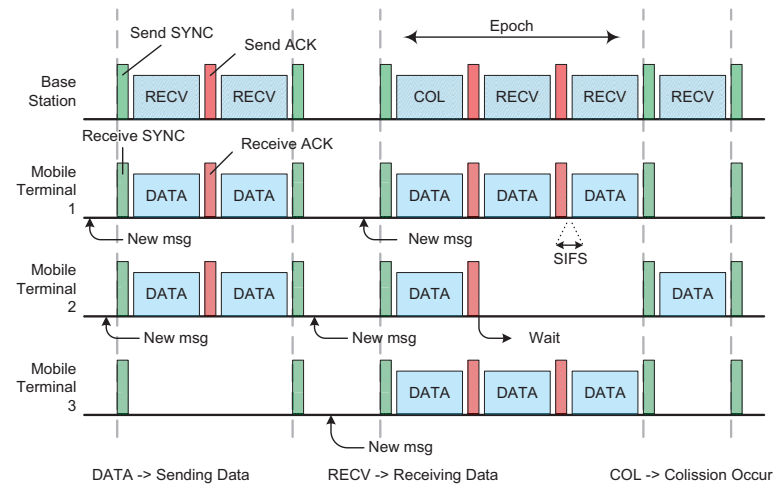

Fig. 1. MAC protocol example.

the half-duplex commutation between sending and receiving mode. No spacing is used between retransmitted packets during the multi-packet detection phase.

Due to the half-duplex approach and the physical and MAC header length, it is not possible to achieve full channel utilization (100\% throughput). For a given $Q_{\max }$ and scheduled traffic, the throughput is maximized if we have $Q_{\max }$ MTs transmitting in each slot, since in this case there are no wasted slots (we are able to detect the $Q_{\max }$ data packets involved in each collision), the number of required ACK and SIFS packets is minimized (only one for the $Q_{\max }$ data packets) and the PER (Packet Error Rate) is minimized (in terms of the energy associated to each transmission attempt and/or the peak energy for each MT). The bound for the half-duplex throughput is given by (1) where, $t_{s i f s}$ is the SIFS duration, $t_{p}$ and $t_{h}$ are the physical preamble and MAC data header duration, and $t_{s y}$ and $t_{a c k}$ are the SYNC and ACK packet payload duration, respectively. Finally, $t_{d a t}$ is the average data packet payload duration.

\section{B. Receiver Structure}

The MAC guarantees that a collision situation of $Q \leq Q_{\max }$ packets is reached. The receiver should then be able to separate the $Q$ packets while performing the FDE procedure. For this purpose we consider the iterative FDE receiver proposed in [12]. However, although the gains of the iterative procedure are very high for large SNR, it only has marginal gains for low SNR, which is the typical working region when we employ channel coding. For this reason, we consider a turbo version of the receiver proposed in [12] where we take advantage of the channel decoder output within the feedback loop.

The time and frequency domain blocks associated to the $q$ th user are $\left\{a_{n, q} ; n=0,1, \ldots, N-1\right\}$ and $\left\{A_{k, q} ; k=\right.$ $0,1, \ldots, N-1\}$, respectively, with $N$ denoting the FFT size. As with other FDE schemes, a suitable cyclic prefix is appended to each transmitted block and removed at the receiver. The resulting signal is passed to the frequency-domain, leading to the frequency-domain blocks associated to the $r$ th version of the collision of $Q$ packets $\left\{Y_{k}^{(r)} ; k=0,1, \ldots, N-1\right\}$, with $Y_{k}^{(r)}=\sum_{q=1}^{Q} A_{k, q}^{(r)} H_{k, q}^{(r)}+N_{k}^{(r)}$, where $H_{k, q}^{(r)}$ denotes the equivalent channel frequency response for the $k$ th subcarrier, the $q$ th user and the $r$ th version of the collision and $N_{k}^{(r)}$ denotes the channel noise. As in [12], we can cope with fixed channels for different versions of the collision (that would produce correlated versions) by performing a cyclic shift on the corresponding transmitted frequency-domain blocks, which is formally equivalent to have a cyclic shift on the corresponding channel.

Let us consider the $i$ th iteration for the detection of the $p$ th packet. If we assume QPSK constellations the channel decoder uses the soft values of the coded bits which are associated with the real and imaginary parts of the time-domain samples ${ }^{3}$ $\left\{\tilde{a}_{n, q}^{(i)} ; n=0,1, \ldots, N-1\right\}=\operatorname{IDFT}\left\{\tilde{A}_{k, q}^{(i)} ; k=0,1, \ldots, N-\right.$ $1\}$, where $\tilde{A}_{k, q}^{(i)}$ denotes the $k$ th frequency-domain sample associated to the $q$ th packet, given by

$$
\tilde{A}_{k, q}^{(i)}=\sum_{r=1}^{Q} F_{k, q}^{(r, i)} Y_{k}^{(r)}-\sum_{q^{\prime}=1}^{Q} B_{k, q}^{\left(q^{\prime}, i\right)} \bar{A}_{k, q^{\prime}}^{\left(i-u\left(q^{\prime}-q\right)\right)}
$$

where $u(x)$ is the unitary step function, i.e., $u(x)=0$ for $x<0$ and 1 for $x \geq 0$. The feedback coefficients $B_{k, q}^{\left(q^{\prime}, i\right)}$ are used to remove the interference between the $q^{\prime}$ packet (for $q^{\prime}=q$ they remove the residual inter-symbol interference) and the feedforward coefficients $F_{k, q}^{(r, i)}$ are used to minimize the residual interference. The feedforward and feedback coefficients are computed as described in [12]. In (2), $\bar{A}_{k, q^{\prime}}^{(i)}$ denotes the average values of $A_{k, q}$ conditioned to the channel decoder output that can be computed as described in [8], [31].

\section{Performance Analysis and Optimization}

This section evaluates the system throughput and delay for homogeneous Poisson traffic, on a wireless network with $J$ MTs. Its main objective is to obtain an analytical model for the behavior of the MAC protocol on a multi-packet reception system, considering different approaches for backoff algorithms as a function of the PER associated to different MTs so as to allow its optimization. In a real system this means that we should be able to estimate the PER fluctuations under given transmission conditions [32], [33] (fortunately, the PER fluctuations are usually relatively slow for the SC-FDE schemes considered in this paper, provided that we have rich multipath propagation).

To achieve this objective we start by calculating the expected epoch duration and the expected number of bytes transmitted per MT and per epoch, to compute the average throughput. After that, we model the MAC behavior employing Markov Chain theory to get the system's steady state. Finally, we characterize the packet service time and identify the throughput-optimal and delay-optimal $p_{c}$ transmission probabilities for the cases when the system is saturated or unsaturated, respectively.

\section{A. Detection process}

Due to the serial structure of the detector [12], the Packet Error Rate $\left(P E R_{Q, q}\right)$ is influenced by the number of sending MTs $(Q)$ and the $q$ th MT. Note that in this model, we consider that MTs are always handled in the same order (i.e., the $q$ th MT is always the $q$ th to be decoded). We also assume that no errors occur in the downlink broadcast transmissions.

\footnotetext{
${ }^{3}$ Actually, in practical implementations the coded bits are interleaved before being mapped into the constellation symbols, which means that the soft values of the coded bits need to be de-interleaved before the decoding procedure.
} 


$$
S \leq \frac{Q_{\text {max }} t_{d a t}}{\left(t_{p}+t_{s y}+t_{s i f s}\right)+\left(t_{p}+t_{a c k}+t_{s i f s}\right)+\left(Q_{\max }\left(t_{p}+t_{h}+t_{d a t}\right)+2 t_{s i f s}\right)}
$$

Let $\psi(Q)$ denote the random variable associated with the number of packets correctly received when $Q$ MTs transmit during the decoding process. The probability of having $k$ packets successfully received $(P\{\psi(Q)=k\})$ can be calculated identifying the set with all error patterns that lead to $k$ packets received, denoted by $\Omega_{Q, k}$. This set is composed by $\left(\begin{array}{l}Q \\ k\end{array}\right)$ members (all the combinations of $Q$ packets with $k$ successes and $Q-k$ errors), each identifying an error pattern. The outcome of the detection process for the packet from the $q$ th MT is an on-off random variable, $\omega_{Q, q}$, which can be 1 (success) or 0 (failure). Let $\omega_{Q}=\left\{\omega_{Q, q} ; q=1, \ldots, Q\right\}$ denote an error pattern, defined by the outcome of all $Q$ packets involved in a collision. The set $\Omega_{Q, k}$ contains all error patterns $\omega_{Q}$ that satisfy the condition $\sum_{q=1}^{Q} \omega_{Q, q}=k$. Therefore, $P\{\psi(Q)=k\}$ can be calculated summing the probability of all error patterns within $\Omega_{Q, k}$,

$$
\begin{aligned}
& P\{\psi(Q)=k\}= \\
& =\sum_{\omega_{Q} \in \Omega_{Q, k}} \prod_{q=1}^{Q}\left(1-P E R_{Q, q}\right)^{\omega_{Q, q}}\left(P E R_{Q, q}\right)^{\left(1-\omega_{Q, q}\right)} .
\end{aligned}
$$

When $Q$ MTs transmit, the expected number of packets jointly detected by the BS is

$$
\theta(Q)=E[\psi(Q)]=\sum_{k=1}^{Q} k P\{\psi(Q)=k\} .
$$

The equation above can be simplified considering the MT's average packet error rate for all $q$, denoted by $P E R_{Q}$, valid for a low variation of $P E R_{Q, q}$ with $q$. Since errors become independent of the decoding order, $\psi(Q)$ has a binomial distribution. In result, the calculation of $\theta(Q)$ is simplified to

$$
\theta(Q)= \begin{cases}\sum_{k=1}^{Q} b i\left(Q, k, 1-P E R_{Q}\right) k, & 1 \leq Q \leq Q_{\max } \\ 0, & Q>Q_{\max }, Q=0\end{cases}
$$

where $b i(Q, k, p)=\left(\begin{array}{c}Q \\ k\end{array}\right) p^{k}(1-p)^{(Q-k)}$ is the binomial probability mass function.

The duration of a group of detection slots, denoted by $\delta(Q)$, measures the duration of the packets exchanged and the short inter-frame space (SIFS) that allows changing the radio between transmitting and receiving modes, $t_{\text {sifs }}$. If $Q$ is zero or above $Q_{\max }$ (the maximum number of detectable transmissions) the detection process fails and lasts a single slot. Otherwise, it lasts $Q$ slots. A group of detection slots always includes an initial contention slot defined by a SYNC or an ACK packets (both packets have the same duration, $t_{p}+t_{s y}=t_{p}+t_{a c k}$ ), followed by a data packet time (we assumed a constant duration equal to $t_{p}+t_{h}+t_{d a t}$ ) and two SIFS. A multipacket detection phase includes an additional ACK packet, $Q-1$ packet retransmissions and two more changes between receiving and transmitting modes. $\delta(Q)$ is defined in (6).

\section{B. Epoch}

An epoch may have multiple unintelligible groups of detection slots (with a single slot dimension) before the last group of detection slots. Let $\nu$ denote a random variable (RV) with the number of unintelligible groups of detection slots (hereafter named simply as unintelligible slots). RV $\nu$ can be defined based on another RV $\eta_{c}$, which denotes the number of MTs that transmit a packet during the $c$ th group of detection slots of an epoch. The $\nu$ 's probability mass function can be defined by $P\{\nu=c\}=P\left\{\eta_{c+1} \leq Q_{\max } \mid \eta_{c}>Q_{\max }\right\}$ for $0<c<M_{C}$, or by $P\left\{\nu=M_{C}\right\}=P\left\{\eta_{M_{C}}>Q_{\max }\right\}$. Finally, $P\{\nu=0\}=P\left\{\eta_{1} \leq Q_{\max }\right\}$.

In this subsection it is shown that both RVs, $\nu$ and $\eta_{c}$, can be described as stochastic processes that depend solely on the number of MTs with packets available to transmit in the first slot of an epoch, denoted by RV $\chi$, and by the value of $p_{c}$, the MTs' transmission probability during the $c$ th group of detection slots.

$\mathrm{RV} \eta_{c}$ is defined when the epoch lasts $0<c \leq M_{C}$ or more groups of detection slots (i.e. $\nu \geq c-1$ ). RV $\chi$ and $p_{1}$ influence the number of packets transmitted in the first group of detection slots of an epoch. MTs that persist contending during an epoch, transmit independently with probability $p_{c}$. Therefore $\eta_{c}$ conditional probability mass function can be defined recursively using (7) where the expression for $1<c \leq M_{C}$ is derived from $P\left\{\eta_{c}=Q \mid \nu \geq c-1\right\}(\chi)=$ $P\left\{\eta_{c}=Q \mid \nu \geq c-2\right\}(\chi) / P\{\nu \geq c-1 \mid \nu \geq c-2\}(\chi)$. $P\{\nu \geq c-1 \mid \nu \geq c-2\}(\chi)$ denotes the probability of having an unintelligible slot at the $(c-1)$ th slot of an epoch, and can be calculated as the probability of having more than $Q_{\max }$ MTs transmitting a packet at that slot, i.e.

$$
\begin{aligned}
& P\{\nu \geq c-1 \mid \nu \geq c-2\}(\chi)= \\
& \sum_{Q=Q_{\max }+1}^{J} P\left\{\eta_{c-1}=Q \mid \nu \geq c-2\right\}(\chi) .
\end{aligned}
$$

Clearly, (7) applies only when $P\{\nu \geq c-1 \mid \nu \geq c-2\}>0$ for $c>1$. From (6) and (7), the $m$ th moment for the duration expectation of an epoch with $c$ groups of detection slots is given by (9) with $\delta\left(Q_{\max }+1\right)$ denoting the duration of a group with a unintelligible slot. Limit $(c)$ is equal to $Q_{\max }$ when $1 \leq c<M_{C}$ and it is equal to $J$ for $c=M_{C}$. It takes account of the number of transmitting MTs $(Q)$ that close an epoch: an epoch with $c$ groups of detection slots has $\eta_{c}=$ $Q \leq Q_{\max }$ if $c<M_{C}$; an epoch with $M_{C}$ group of detection slots has $\eta_{M_{C}-1}=Q>Q_{\max }$ and it does not depend on $\eta_{M_{C}}$, because it is the last possible group of detecting slots.

Similarly, the $m$ th moment of the expected number of bytes received during an epoch with $c$ groups of detection slots is

$$
\begin{aligned}
& \operatorname{num}_{c}^{m}(\chi)= \\
& =\sum_{Q=0}^{\operatorname{Limit}(c)}\left(L_{\text {data }} \theta(Q)\right)^{m} P\left\{\eta_{c}=Q \mid \nu \geq c-1\right\}(\chi),
\end{aligned}
$$

where $\theta(Q)$, defined by (5), accounts for the successful detection rate.

The probability of occurring at least $c-1$ unintelligible slots during an epoch is calculated using (11). 